Research Article

\title{
Strong Convergence Theorems for Asymptotically Weak G-Pseudo- $\Psi$-Contractive Non-Self-Mappings with the Generalized Projection in Banach Spaces
}

\author{
Yuanheng Wang \\ Department of Mathematics, Zhejiang Normal University, Zhejiang 321004, China \\ Correspondence should be addressed to Yuanheng Wang,wangyuanheng@yahoo.com.cn \\ Received 28 August 2012; Revised 7 October 2012; Accepted 9 October 2012 \\ Academic Editor: Xiaolong Qin
}

Copyright (c) 2012 Yuanheng Wang. This is an open access article distributed under the Creative Commons Attribution License, which permits unrestricted use, distribution, and reproduction in any medium, provided the original work is properly cited.

A new concept of the asymptotically weak $G$-pseudo- $\Psi$-contractive non-self-mapping $T: G \mapsto B$ is introduced and some strong convergence theorems for the mapping are proved by using the generalized projection method combined with the modified successive approximation method or with the modified Mann iterative sequence method in a uniformly and smooth Banach space. The proof methods are also different from some past common methods.

\section{Introduction}

Let $B$ be a real Banach space with the norm $\|\cdot\|, B^{*}$ its dual space with the norm $\|\cdot\|_{*}$. As usually, we introduce a dual product in $B^{*} \times B$ by $\left\langle x^{*}, x\right\rangle$, where $x^{*} \in B^{*}$ and $x \in B$. Let $J: B \mapsto B^{*}$ be the normalized duality mapping $J$ in $B$ defined as

$$
J x=\left\{f \in B^{*}:\langle f, x\rangle=\|f\|_{*}\|x\|=\|x\|^{2}\right\}, \quad \forall x \in B
$$

It is clear that the operator $J$ is well defined in a Banach space by the famous Hahn-Banach theorem.

The concept of asymptotically nonexpansive mappings was first introduced by Goebel and Kirk [1] in 1972 and then Schu [2] introduced the asymptotically pseudocontractive mappings in 1991.

Definition 1.1. Let $G$ be a nonempty subset of a real Banach space $B$ and $T: G \mapsto G$ be a mapping. 
(1) The mapping $T$ is said to be asymptotically nonexpansive, if there exists a number sequence $\left\{k_{n}\right\}$ in $[1, \infty)$ with $\lim _{n \rightarrow \infty} k_{n}=1$ such that

$$
\left\|T^{n} x-T^{n} y\right\| \leq k_{n}\|x-y\|,
$$

for all $x, y \in G$ and $n \geq 1$.

(2) The mapping $T$ is said to be asymptotically pseudocontractive, if for all $x, y \in G$, there exists a number sequence $\left\{k_{n}\right\}$ in $[1, \infty)$ with $\lim _{n \rightarrow \infty} k_{n}=1$ and $j(x-y) \in$ $J(x-y)$ such that

$$
\left\langle T^{n} x-T^{n} y, j(x-y)\right\rangle \leq k_{n}\|x-y\|^{2} .
$$

(3) The mapping $T$ is said to be asymptotically demi-pseudocontractive, if for all $x \in G$, $p \in F(T)$, there exists a number sequence $k_{n}$ in $[1, \infty)$ with $\lim _{n \rightarrow \infty} k_{n}=1$ and $j(x-y) \in J(x-y)$ such that

$$
\left\langle T^{n} x-p, j(x-p)\right\rangle \leq k_{n}\|x-p\|^{2},
$$

where $F(T) \neq \emptyset$ is the set of all fixed points of the mapping $T$.

The iterative approximation problems for asymptotically nonexpansive and pseudocontractive mapping $T$ were studied by many authors and we always assume that the fixed point set $F(T)$ of the operator $T$ is nonempty, such as see [1-6]. In 2011, Qin et al. [7] introduced a new concept of the asymptotically strict quasi- $\Phi$-pseudocontractive mapping $T: G \rightarrow G$. They combined the generalized projection $\Pi_{G}$ to give a new iterative sequence for the $T$ and proved that the sequence converges strongly to a point $x^{\prime}=\Pi_{F(T)} x_{0}$.

But, all these arguments are not enough if the operator $T$ acts from $G$ to $B$, which we called non-self-mappings, and the iterative methods we used to be, such as Mann iterative method and its some modifications, can not be used. Under this condition, it is natural for us to try to consider the metric projection operator $P_{G}: B \mapsto G$ and the generalized projection operator $\Pi_{G}: B \mapsto G$, and some authors have given relevant results and applications of the operator $P_{G}$ and $\pi_{G}$ (see [8-11]).

Very recently, in 2012, Yao et al. [12] and Liou et al. [13] considered the non-selfmapping $T: G \subseteq H \rightarrow H$ in a Hilbert space $H$. They also proved their new iterative sequence for the $T$ combined with the metric projection $P_{G}$ converges strongly to a point $x^{\prime}=P_{V I(G, T)}(0)$ and the unique solution of a variational inequality, respectively.

Motivated and inspired by the said above, we first introduce a new concept of the asymptotically weak $G$-pseudo- $\Psi$-contractive non-self-mapping $T: G \mapsto B$. Then, in a uniformly convex and smooth Banach space, we prove some strong convergence theorems for the mapping by using the generalized projection method and the modified successive approximation method

$$
x_{n+1}=\left(\Pi_{G} T\right)^{n} x_{n}, \quad n=1,2, \ldots, x_{1} \in G
$$


or the modified Mann iterative sequence method

$$
x_{n+1}=Q_{G}\left(\left(1-\alpha_{n}\right) x_{n}+\alpha_{n} T\left(\Pi_{G} T\right)^{n-1} x_{n}\right), \quad n=1,2, \ldots, x_{1} \in G,
$$

where $Q_{G}: B \mapsto G$ is a sunny nonexpansive retraction. So, in some ways, our results extend and improve some results of other authors (such as, see [1-5, 7, 9-13]), from self mappings to non-self-mappings, from Hilbert spaces to Banach spaces.

\section{Preliminaries}

In the sequel, we will assume that $B$ is a real uniformly convex and uniformly smooth (hence reflexive) Banach space, then $B^{*}$ will be the same. If we denote by $\delta_{B}(\varepsilon)$ the modulus of convexity of the Banach space $B$ and by $\rho_{B}(\tau)$ its modulus of smoothness, then

$$
\delta_{B}(\varepsilon), \rho_{B}(\tau), g_{B}(\varepsilon)=\varepsilon^{-1} \delta_{B}(\varepsilon), \quad h_{B}(\tau)=\tau^{-1} \rho_{B}(\tau)
$$

are all continuous and increasing on their domains, respectively, and $\delta_{B}(0)=\rho_{B}(0)=g_{B}(0)=$ $h_{B}(0)=0$ (see [9]). Also, under the conditions the normalized duality operator

$$
J: J x=\frac{1}{2} \operatorname{grad}\left\{\|x\|^{2}\right\}
$$

is single-valued, strictly monotone, continuous, coercive, bounded, and homogeneous, but not addible. In a Hilbert space, $J$ is the Identity operator $I: I x=x$.

Definition 2.1 (see $[10,11]$ ). The operator $P_{G}: B \mapsto G \subseteq B$ is called metric projection operator if it assigns to each $x \in B$ its nearest point $\bar{x} \in G$, that is, the solution $\bar{x}$ for the minimization problem

$$
P_{G} x=\bar{x} ; \quad \bar{x}:\|x-\bar{x}\|=\inf _{\xi \in G}\|x-\xi\| .
$$

The operator $\Pi_{G}: B \mapsto G \subseteq B$ is called the generalized projection operator if it assigns to each $x \in B$ a minimum point $\widehat{x} \in G$ of the Lapunov function $V(x, \xi): B \times B \mapsto[0, \infty)$ :

$$
V(x, \xi)=\|x\|^{2}-2\langle J x, \xi\rangle+\|\xi\|^{2},
$$

that is, a solution of the following minimization problem:

$$
\Pi_{G} x=\widehat{x} ; \quad \widehat{x}: V(x, \widehat{x})=\inf _{\xi \in G} V(x, \xi) .
$$

Lemma 2.2 (see $[10,11]$ ). The point $\bar{x}=P_{G} x$ is the metric projection of $x \in B$ on $G \subseteq B$ if and only if the following inequality is satisfied:

$$
\langle J(x-\bar{x}), \bar{x}-\xi\rangle \geq 0, \quad \forall \xi \in G,
$$

and the operator $P_{G}$ is nonexpansive in Hilbert spaces. 
The point $\widehat{x}=\Pi_{G} x$ is the generalized projection of $x \in B$ on $G \subseteq B$ if and only if the following inequality is satisfied:

$$
\langle J x-J \widehat{x}, \widehat{x}-\xi\rangle \geq 0, \quad \forall \xi \in G
$$

Furthermore, the inequality below also holds:

$$
V\left(\Pi_{G} x, \xi\right) \leq V(x, \xi)-V\left(x, \Pi_{G} x\right), \quad \forall \xi \in G
$$

And thus, we have

$$
V\left(\Pi_{G} x, \xi\right) \leq V(x, \xi), \quad \forall \xi \in G
$$

Lemma 2.3 (see [8]). For all $x, y \in B$, if $\|x\| \leq R$ and $\|y\| \leq R$, then the following inequality is satisfied:

$$
(2 L)^{-1} R^{2} \delta_{B}\left(\frac{\|x-y\|}{4 R}\right) \leq V(x, y) \leq 4 L R^{2} \rho_{B}\left(\frac{4\|x-y\|}{R}\right)
$$

where $L: 1<L<1.7$ is a constant.

In general, the operator $P_{G}$ and $\Pi_{G}$ are not nonexpansive in Banach spaces. It is easy to see $P_{G}=\Pi_{G}$ in Hilbert spaces because of $J=I$. In a uniformly convex and uniformly smooth Banach space, $P_{G}$ is well defined on a closed convex set $G$ and $\Pi_{G}$ is also well defined on a closed convex set $G$ from the properties of the functional $V(x, \xi)$ and strict monotonicity of the mapping $J$. More properties of the mappings $J, V, P_{G}$, and $\Pi_{G}$ and some of their applications can be found in [8-11].

Definition 2.4 (see [14]). Let $B$ be a real Banach space, $G \subseteq B$ be a subset. The operator $Q_{G}$ : $B \mapsto G$ is called sunny nonexpansive retract if $Q_{G}$ is nonexpansive, $Q_{G}^{2}=Q_{G}$, and for any $x \in G, t>0, t x+(1-t) Q_{G} x \in G$ holds $Q_{G}\left(t x+(1-t) Q_{G} x\right)=Q_{G} x$.

If $B$ is a uniformly smooth Banach space and $G \subset B$, is a closed convex set, then the unique sunny nonexpansive retract $Q_{G}$ exists.

Definition 2.5. Let $B$ be a real Banach space, $G$ be a nonempty subset of $B$, and $T: G \mapsto B$ be a non-self-mapping. If there exists a sequence $\left\{k_{n}\right\}$ in $[1, \infty)$ with $\lim _{n \rightarrow \infty} k_{n}=1$ and a continuous increasing function $\Psi(t)$ for all $t>0$ with $\Psi(0)=0, \lim _{t \rightarrow \infty} \Psi(t)=\infty$, it is shown as follows, respectively:

(1) The mapping $T$ is said to be asymptotically weak $G$ - $\Psi$-contractive mapping, if

$$
V\left(T\left(\Pi_{G} T\right)^{n-1} x, T\left(\Pi_{G} T\right)^{n-1} y\right) \leq k_{n} V(x, y)-\Psi(V(x, y)) .
$$


(2) The mapping $T$ is said to be asymptotically weak G-quasi- $\Psi$-contractive mapping, if

$$
V\left(T\left(\Pi_{G} T\right)^{n-1} x, x^{*}\right) \leq k_{n} V\left(x, x^{*}\right)-\Psi\left(V\left(x, x^{*}\right)\right)
$$

for all $x, y \in G, x^{*} \in F(T)$, and $n \geq 1$, where $F(T) \neq \emptyset$.

(3) The mapping $T$ is said to be asymptotically weak $G$ - $\Psi$-pseudocontractive mapping, if

$$
\left\langle T\left(\Pi_{G} T\right)^{n-1} x-T\left(\Pi_{G} T\right)^{n-1} y, j(x-y)\right\rangle \leq k_{n}\|x-y\|^{2}-\Psi\left(\|x-y\|^{2}\right),
$$

for all $x, y \in G, j(x-y) \in J(x-y)$.

(4) The mapping $T$ is said to be asymptotically weak G-quasi- $\Psi$-pseudocontractive mapping, if

$$
\left\langle T\left(\Pi_{G} T\right)^{n-1} x-x^{*}, j\left(x-x^{*}\right)\right\rangle \leq k_{n}\left\|x-x^{*}\right\|^{2}-\Psi\left(\left\|x-x^{*}\right\|^{2}\right),
$$

for all $x, y \in G, x^{*} \in F(T), j\left(x-x^{*}\right) \in J\left(x-x^{*}\right)$, and $n \geq 1$, where $F(T) \neq \emptyset$.

Remark 2.6. It is clear that one can omit each operator $\Pi_{G}$ in (2.11)-(2.14) if the mapping $T$ acts from $G$ to $G$, that is, $T: G \mapsto G \subseteq B$ is a self-mapping. So, the class of asymptotically weak $G-\Psi$-contractive mappings contains that of asymptotically nonexpansive mappings and the class of asymptotically weak $G-\Psi$-pseudocontractive mappings contains that of asymptotically pseudocontractive mappings. Therefore, all the results and applications of asymptotically nonexpansive mappings can be as a part of the asymptotically weak G- $\Psi$ contractive mappings.

In order to prove our main results, we also need the following lemmas.

Lemma 2.7 (see [15]). Let $\left\{\lambda_{n}\right\},\left\{\alpha_{n}\right\},\left\{\beta_{n}\right\}$, and $\left\{\gamma_{n}\right\}$ be sequences of nonnegative numbers satisfying the following conditions:

$$
\alpha_{n}>0, \quad \sum_{n=1}^{\infty} \alpha_{n}=\infty, \quad \sum_{n=1}^{\infty} \beta_{n}<\infty, \quad \lim _{n \rightarrow \infty} \frac{\gamma_{n}}{\alpha_{n}}=0
$$

Suppose the following recurse inequality holds:

$$
\lambda_{n+1} \leq\left(1+\beta_{n}\right) \lambda_{n}-\alpha_{n} \psi\left(\lambda_{n}\right)+\gamma_{n}, \quad n=1,2, \ldots,
$$

where $\psi(t)$ is a continuous strictly increasing function for all $t>0$ with $\psi(0)=0, \lim _{t \rightarrow \infty} \psi(t)=\infty$. Then $\lambda_{n} \rightarrow 0$ as $n \rightarrow \infty$. 
Lemma 2.8 (see [16]). Let B be a real Banach space and $J$ be the normalized duality mapping. Then

$$
\|x+y\|^{2} \leq\|x\|^{2}+2\langle y, j(x+y)\rangle
$$

for all $x, y \in B$ and $j(x+y) \in J(x+y)$.

\section{Main Results}

Theorem 3.1. Let $B$ be a uniformly convex and uniformly smooth Banach space, $G$ be a closed convex subset of $B, T: G \mapsto B$ be an asymptotically weak $G$-quasi- $\Psi$-contractive mapping with a sequence $\left\{k_{n}\right\} \subseteq[1, \infty), \sum_{n=1}^{\infty}\left(k_{n}-1\right)<\infty$, and $x^{*} \in G$ is its fixed point. Then the iterative sequence $\left\{x_{n}\right\}$ generated by the modified successive approximation method (1.5) is bounded for all $n \geq 1$ and converges strongly to $x^{*}$.

Proof. If $x^{*} \in F(T)$ is the fixed point of $T$ in $G$, that is, $T x^{*}=x^{*}$, then we get by (1.5) and (2.8) in Lemma 2.2 for $x^{*} \in G$,

$$
\begin{aligned}
V\left(x_{n+1}, x^{*}\right)=V\left(\left(\Pi_{G} T\right)^{n} x_{n}, x^{*}\right) & =V\left(\Pi_{G} T\left(\Pi_{G} T\right)^{n-1} x_{n}, x^{*}\right) \\
& \leq V\left(T\left(\Pi_{G} T\right)^{n-1} x_{n}, x^{*}\right) .
\end{aligned}
$$

We use the condition (2.12) of asymptotically weak G-quasi- $\Psi$-contractive of the operator $T$ and get

$$
\begin{aligned}
V\left(x_{n+1}, x^{*}\right) & \leq k_{n} V\left(x_{n}, x^{*}\right)-\psi\left(V\left(x_{n}, x^{*}\right)\right) \leq k_{n} V\left(x_{n}, x^{*}\right) \\
& \leq k_{n} k_{n-1} V\left(x_{n-1}, x^{*}\right) \leq \cdots \leq k_{n} k_{n-1} \cdots k_{1} V_{1}\left(x_{1}, x^{*}\right) .
\end{aligned}
$$

Because $\sum_{n=1}^{\infty}\left(k_{n}-1\right)<\infty$, we know $\lim _{n \rightarrow \infty} k_{n} k_{n-1} \cdots k_{1}=$ constant and $\left\{k_{n} k_{n-1} \cdots k_{1}\right\}$ is bounded, say by $K: 1 \leq k_{n} k_{n-1} \cdots k_{1} \leq K$ for all $n \geq 1$.

It is obviously that $V(x, \xi)=\|x\|^{2}-2\langle J x, \xi\rangle+\|\xi\|^{2}$ satisfies the inequality

$$
(\|x\|-\|\xi\|)^{2} \leq V(x, \xi) \leq(\|x\|+\|\xi\|)^{2} .
$$

Therefore by (3.2) and (3.3), we have

$$
\begin{aligned}
& \left\|x_{n}\right\|-\left\|x^{*}\right\| \leq \sqrt{k_{n} k_{n-1} \cdots k_{1} V_{1}\left(x_{1}, x^{*}\right)} \leq \sqrt{K}\left(\left\|x_{1}\right\|+\left\|x^{*}\right\|\right), \\
& \left\|x_{n}\right\| \leq \sqrt{K}\left\|x_{1}\right\|+(1+\sqrt{K})\left\|x^{*}\right\|,
\end{aligned}
$$

for all $n \geq 1$, that is, the sequence $\left\{x_{n}\right\}$ is bounded.

The sequence of positive number $\left\{\lambda_{n}\right\}$ defined by $\lambda_{n}=V\left(x_{n}, x^{*}\right)$ are bounded and from (3.2) it satisfies the following inequality:

$$
\lambda_{n+1} \leq\left(1+\beta_{n}\right) \lambda_{n}-\alpha_{n} \Psi\left(\lambda_{n}\right)+\gamma_{n}, \quad n=1,2, \ldots
$$


where $\beta_{n}=k_{n}-1, \sum_{n=1}^{\infty} \beta_{n}<\infty, \alpha_{n}=1, \sum_{n=1}^{\infty} \alpha_{n}=\infty, \gamma_{n}=0$. So, using Lemma 2.7 we get

$$
\lim _{n \rightarrow \infty} V\left(x_{n}, x^{*}\right)=0
$$

Because $R=\sqrt{K}\left\|x_{1}\right\|+(1+\sqrt{K})\left\|x^{*}\right\|$ is a constant and $\left\|x_{n}\right\| \leq R,\left\|y_{n}\right\| \leq R$, we obtain from the left part of the estimate of (2.10) in Lemma 2.3 the following:

$$
0 \leq \lim _{n \rightarrow \infty} 2 L^{-1} R^{2} \delta_{B}\left(\frac{\left\|x_{n}-x^{*}\right\|}{4 R}\right) \leq \lim _{n \rightarrow \infty} V\left(x_{n}, x^{*}\right)=0 .
$$

By the properties of $\delta_{B}(\varepsilon)$, this implies

$$
\lim _{n \rightarrow \infty}\left\|x_{n}-x^{*}\right\|=0
$$

that is, the sequence $\left\{x_{n}\right\}$ converges strongly to fixed point $x^{*}$.

Corollary 3.2. Let $G$ be a closed convex set in $B, T: G \mapsto B$ be an asymptotically weak $G-\Psi$ contractive mapping with a sequence $\left\{k_{n}\right\} \subseteq[1, \infty), \sum_{n=1}^{\infty}\left(k_{n}-1\right)<\infty$, and $x^{*} \in G$ its fixed point. Then the iterative sequence $\left\{x_{n}\right\}$ defined by modified successive approximation method (1.5) converges strongly to $x^{*}$.

Proof. If we take $y=x^{*} \in G$ as the fixed point of $T$, then we have

$$
\begin{aligned}
T\left(\pi_{G} T\right)^{n-1} x^{*} & =T\left(\pi_{G} T\right)^{n-2}\left(\pi_{G} T x^{*}\right)=T\left(\pi_{G} T\right)^{n-2}\left(\pi_{G} x^{*}\right) \\
& =T\left(\pi_{G} T\right)^{n-2} x^{*}=\cdots=T x^{*}=x^{*} .
\end{aligned}
$$

So the asymptotically weak $G$ - $\Psi$-contractive mapping $T: G \mapsto B$ is also an asymptotically weak G-quasi- $\Psi$-contractive mapping and the results of Theorem 3.1 still hold.

Theorem 3.3. Let $B$ be a real uniformly convex and uniformly smooth Banach space, $G$ be a nonempty closed convex subset of $B, T: G \mapsto B$ be an asymptotically weak G-quasi- $\Psi$-pseudocontractive mapping with a sequence $\left\{k_{n}\right\} \subseteq[1, \infty), \sum_{n=1}^{\infty}\left(k_{n}-1\right)<\infty$, and $x^{*} \in G$ its fixed point. Consider the iterative sequence $\left\{x_{n}\right\}$ defined by the modified Mann iterative sequence method (1.6). Suppose the sequence $\left\{x_{n}\right\}$ and $\left\{T\left(\Pi_{G} T\right)^{n-1} x_{n}\right\}$ are bounded, $\left\{\alpha_{n}\right\}$ is a number sequence in $(0,1]$ satisfing the conditions below:

$$
\sum_{n=1}^{\infty} \alpha_{n}=\infty, \quad \sum_{n=1}^{\infty} \alpha_{n}^{2}<\infty
$$

where $Q_{G}: B \mapsto G$ is a sunny nonexpansive retraction. Then the iterative sequence $\left\{x_{n}\right\}$ converges strongly to $x^{*}$. 
Proof. By the virtue of (2.17) in Lemma 2.8, it follows that

$$
\begin{aligned}
\left\|x_{n+1}-x^{*}\right\|^{2}= & \left\|Q_{G}\left(\left(1-\alpha_{n}\right) x_{n}+\alpha_{n} T\left(\Pi_{G} T\right)^{n-1} x_{n}\right)-Q_{G} x^{*}\right\|^{2} \\
\leq & \left\|\left(1-\alpha_{n}\right) x_{n}+\alpha_{n} T\left(\Pi_{G} T\right)^{n-1} x_{n}-x^{*}\right\|^{2} \\
& =\left\|\left(1-\alpha_{n}\right)\left(x_{n}-x^{*}\right)+\alpha_{n}\left(T\left(\Pi_{G} T\right)^{n-1} x_{n}-x^{*}\right)\right\|^{2} \\
\leq & \left(1-\alpha_{n}\right)^{2}\left\|\left(x_{n}-x^{*}\right)\right\|^{2}+2 \alpha_{n}\left\langle T\left(\Pi_{G} T\right)^{n-1} x_{n}-x^{*}, j\left(x_{n+1}-x^{*}\right)\right\rangle \\
& =\left(1-\alpha_{n}\right)^{2}\left\|\left(x_{n}-x^{*}\right)\right\|^{2}+2 \alpha_{n}\left\langle T\left(\Pi_{G} T\right)^{n-1} x_{n}-x^{*}, j\left(x_{n}-x^{*}\right)\right\rangle \\
& +2 \alpha_{n}\left\langle T\left(\Pi_{G} T\right)^{n-1} x_{n}-x^{*}, j\left(x_{n+1}-x^{*}\right)-j\left(x_{n}-x^{*}\right)\right\rangle .
\end{aligned}
$$

Since $\left\{x_{n}-T\left(P_{G} T\right)^{n-1} x_{n}\right\}$ is bounded, say by $K$, we have

$$
\begin{aligned}
\left\|\left(x_{n+1}-x^{*}\right)-\left(x_{n}-x^{*}\right)\right\| & =\left\|Q_{G}\left(\left(1-\alpha_{n}\right) x_{n}+\alpha_{n} T\left(P_{G} T\right)^{n-1} x_{n}\right)-Q_{G} x_{n}\right\| \\
& \leq\left\|\left(1-\alpha_{n}\right) x_{n}+\alpha_{n} T\left(P_{G} T\right)^{n-1} x_{n}-x_{n}\right\| \\
& =\alpha_{n}\left\|x_{n}-T\left(P_{G} T\right)^{n-1} x_{n}\right\| \\
& \leq \alpha_{n} K .
\end{aligned}
$$

From (3.10) we know $\lim _{n \rightarrow \infty} \alpha_{n}=0$ and then one gets

$$
\lim _{n \rightarrow \infty}\left\|x_{n+1}-x_{n}\right\|=\lim _{n \rightarrow \infty}\left\|\left(x_{n+1}-x^{*}\right)-\left(x_{n}-x^{*}\right)\right\|=0 .
$$

By using the uniform continuity of $j=J$ in the uniformly convex and uniform smooth Banach space $B$ and the bound of the sequence $\left\{T\left(\Pi_{G} T\right)^{n-1} x_{n}-x^{*}\right\}$, we have

$$
\gamma_{n}:=\left\langle T\left(\Pi_{G} T\right)^{n-1} x_{n}-x^{*}, j\left(x_{n+1}-x^{*}\right)-j\left(x_{n}-x^{*}\right)\right\rangle \rightarrow 0, \quad n \rightarrow \infty .
$$


Substituting (3.14) into (3.11) and using (2.14), we get

$$
\begin{aligned}
\| x_{n+1} & -x^{*} \|^{2} \\
& \leq\left(1-\alpha_{n}\right)^{2}\left\|x_{n}-x^{*}\right\|^{2}+2 \alpha_{n}\left\langle T\left(\Pi_{G} T\right)^{n-1} x_{n}-x^{*}, j\left(x_{n}-x^{*}\right)\right\rangle+2 \alpha_{n} \gamma_{n} \\
& \leq\left(1-\alpha_{n}\right)^{2}\left\|x_{n}-x^{*}\right\|^{2}+2 \alpha_{n}\left(k_{n}\left\|x_{n}-x^{*}\right\|^{2}-\Psi\left(\left\|x_{n}-x^{*}\right\|^{2}\right)\right)+2 \alpha_{n} \gamma_{n} \\
& =\left(1+2 \alpha_{n}\left(k_{n}-1\right)+\alpha_{n}^{2}\right)\left\|x_{n}-x^{*}\right\|^{2}-2 \alpha_{n} \Psi\left(\left\|x_{n}-x^{*}\right\|^{2}\right)+2 \alpha_{n} \gamma_{n} \\
& \leq\left(1+2\left(k_{n}-1\right)+\alpha_{n}^{2}\right)\left\|x_{n}-x^{*}\right\|^{2}-2 \alpha_{n} \Psi\left(\left\|x_{n}-x^{*}\right\|^{2}\right)+2 \alpha_{n} \gamma_{n} .
\end{aligned}
$$

Thus, the sequence of positive number $\left\{\lambda_{n}\right\} \underset{n=1}{\infty}$ defined by $\lambda_{n}=\left\|x_{n}-x^{*}\right\|^{2}$ satisfies the recursive inequality

$$
\lambda_{n+1} \leq\left(1+\beta_{n}\right) \lambda_{n}-2 \alpha_{n} \psi\left(\left\|x_{n}-x^{*}\right\|^{2}\right)+2 \alpha_{n} \gamma_{n}
$$

where $\beta_{n}=2\left(k_{n}-1\right)+\alpha_{n}^{2}, \sum_{n=1}^{\infty} \beta_{n}=2 \sum_{n=1}^{\infty}\left(k_{n}-1\right)+\sum_{n=1}^{\infty} \alpha_{n}^{2}<\infty, \sum_{n=1}^{\infty}\left(2 \alpha_{n}\right)=\infty, \gamma_{n} \rightarrow 0$ as $n \rightarrow \infty$. Therefore by the virtue of Lemma 2.7, it is clear that the assertion $\lambda_{n} \rightarrow 0$ as $n \rightarrow \infty$ holds, that is,

$$
\lim _{n \rightarrow \infty}\left\|x_{n}-x^{*}\right\|^{2}=0, \quad \lim _{n \rightarrow \infty}\left\|x_{n}-x^{*}\right\|=0
$$

Corollary 3.4. Let $B$ be a real uniformly convex and uniformly smooth Banach space, $G$ be a nonempty closed convex subset of $B, T: G \mapsto B$ be an asymptotically weak $G$ - $\Psi$-pseudocontractive mapping with a sequence $\left\{k_{n}\right\} \subseteq[1, \infty), \sum_{n=1}^{\infty}\left(k_{n}-1\right)<\infty$, and $x^{*} \in G$ its fixed point. Consider the iterative sequence $\left\{x_{n}\right\}$ defined by the modified Mann iterative sequence (1.6). Suppose the sequence $\left\{x_{n}\right\}$ and $\left\{T\left(\Pi_{G} T\right)^{n-1} x_{n}\right\}$ are bounded, $\left\{\alpha_{n}\right\}$ is a real number sequence in $(0,1]$ satisfying the conditions (3.10). Then one has

$$
\lim _{n \rightarrow \infty}\left\|x_{n+1}-x_{n}\right\|=0
$$

and the iterative sequence $\left\{x_{n}\right\}$ converges in norm to $x^{*}$.

Proof. Following Theorem 3.3, we can have the assertions of the corollary.

Remark 3.5. Because a Hilbert space must be a uniformly convex and uniformly smooth Banach space, the above results still hold in a Hilbert space. In fact, if we notice $\Pi_{G}=P_{G}$ in Hilbert spaces, we can abate some conditions in Corollary 3.4 and have the following theorem. 
Theorem 3.6. Let $G$ be a closed convex set of a Hilbert space $H . T: G \mapsto H$ is said to be

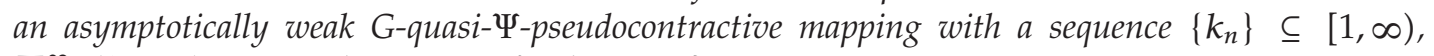
$\sum_{n=1}^{\infty}\left(k_{n}-1\right)<\infty$, and $x^{*} \in G$ its fixed point, if

$$
\left\|T\left(P_{G} T\right)^{n-1} x-x^{*}\right\| \leq k_{n}\left\|x-x^{*}\right\|-\psi\left(\left\|x-x^{*}\right\|\right),
$$

where $\Psi$ is a continuous increasing function for all $t>0$ with $\Psi(0)=0, \lim _{t \rightarrow \infty} \Psi(t)=\infty$. Consider the new modified Mann iterative sequence $\left\{x_{n}\right\}$ defined by the modified Mann iterative sequence (1.6). If the number sequence $\left\{\alpha_{n}\right\}$ satisfies the conditions

$$
0<\alpha_{n} \leq 1, \quad \sum_{n=1}^{\infty} \alpha_{n}=\infty
$$

then the iterative sequence $\left\{x_{n}\right\}$ converges strongly to $x^{*}$.

Proof. Because $P_{G}=\Pi_{G}$ is nonexpansive in Hilbert spaces, $\left\{\alpha_{n}\right\}$ satisfies (3.20) and the operator $T$ satisfies (3.19), we get

$$
\begin{aligned}
\left\|x_{n+1}-x^{*}\right\| & =\left\|P_{G}\left(\left(1-\alpha_{n}\right) x_{n}+\alpha_{n} T\left(P_{G} T\right)^{n-1} x_{n}\right)-P_{G} x^{*}\right\| \\
& \leq\left\|\left(1-\alpha_{n}\right) x_{n}+\alpha_{n} T\left(P_{G} T\right)^{n-1} x_{n}-x^{*}\right\| \\
& =\left\|\left(1-\alpha_{n}\right)\left(x_{n}-x^{*}\right)+\alpha_{n}\left(T\left(P_{G} T\right)^{n-1} x_{n}-x^{*}\right)\right\| \\
& \leq\left(1-\alpha_{n}\right)\left\|\left(x_{n}-x^{*}\right)\right\|+\alpha_{n}\left\|T\left(P_{G} T\right)^{n-1} x_{n}-x^{*}\right\| \\
& \leq\left(1-\alpha_{n}\right)\left\|\left(x_{n}-x^{*}\right)\right\|+\alpha_{n}\left(k_{n}\left\|\left(x_{n}-x^{*}\right)\right\|-\psi\left(\left\|\left(x_{n}-x^{*}\right)\right\|\right)\right) \\
& =\left(1+\alpha_{n}\left(k_{n}-1\right)\right)\left\|\left(x_{n}-x^{*}\right)\right\|-\alpha_{n} \psi\left(\left\|\left(x_{n}-x^{*}\right)\right\|\right) \\
& \leq k_{n}\left\|\left(x_{n}-x^{*}\right)\right\|-\alpha_{n} \psi\left(\left\|\left(x_{n}-x^{*}\right)\right\|\right) .
\end{aligned}
$$

Denote $\lambda_{n}=\left\|x_{n}-x^{*}\right\|$ and we have the following inequality:

$$
\lambda_{n+1} \leq\left(1+\beta_{n}\right) \lambda_{n}-\alpha_{n} \psi\left(\lambda_{n}\right)+\gamma_{n}
$$

where $\beta_{n}=k_{n}-1, \sum_{n=1}^{\infty} \beta_{n}<\infty, \alpha_{n} \in(0,1], \sum_{n=1}^{\infty} \alpha_{n}=\infty, \gamma_{n}=0$. Therefore we know $\lambda_{n} \rightarrow 0$ as $n \rightarrow \infty$ by using Lemma 2.7 , that is,

$$
\lim _{n \rightarrow \infty}\left\|x_{n}-x^{*}\right\|=0
$$

Remark 3.7. It is clear that the above results, in some ways, extend and improve some results of other authors (such as, see [1-5, 7, 9-13]), from self mappings to non-self-mappings, from 
Hilbert spaces to Banach spaces. And in the proof process, our methods are different from some past common methods.

\section{Acknowledgments}

The authors would like to thank editors and referees for many useful comments and suggestions for the improvement of the paper. This work was partially supported by the Natural Science Foundation of Zhejiang Province (Y6100696) and the National Natural Science Foundation of China $(11071169,11271330)$.

\section{References}

[1] K. Goebel and W. A. Kirk, "A fixed point theorem for asymptotically nonexpansive mappings," Proceedings of the American Mathematical Society, vol. 35, pp. 171-174, 1972.

[2] J. Schu, "Iterative construction of fixed points of asymptotically nonexpansive mappings," Journal of Mathematical Analysis and Applications, vol. 158, no. 2, pp. 407-413, 1991.

[3] X. Qin, Y. Su, and M. Shang, "Strong convergence for three classes of uniformly equi-continuous and asymptotically quasi-nonexpansive mappings," Journal of the Korean Mathematical Society, vol. 45, no. 1, pp. 29-40, 2008.

[4] B. E. Rhoades, "Convergence of an Ishikawa-type iteration scheme for a generalized contraction," Journal of Mathematical Analysis and Applications, vol. 185, no. 2, pp. 350-355, 1994.

[5] K.-K. Tan and H. K. Xu, "Fixed point iteration processes for asymptotically nonexpansive mappings," Proceedings of the American Mathematical Society, vol. 122, no. 3, pp. 733-739, 1994.

[6] S. S. Chang, "Some results for asymptotically pseudo-contractive mappings and asymptotically nonexpansive mappings," Proceedings of the American Mathematical Society, vol. 129, no. 3, pp. 845853, 2001.

[7] X. Qin, T. Wang, and S. Y. Cho, "Hybrid projection algorithms for asymptotically strict quasi- $\phi$ pseudocontractions," Abstract and Applied Analysis, vol. 2011, Article ID 142626, 13 pages, 2011.

[8] Y. I. Alber, "Metric and generalized projection operators in Banach spaces: properties and applications," in Theory and Applications of Nonlinear Operators of Accretive and Monotone Type, vol. 178 of Lecture Notes in Pure and Applied Mathematics, pp. 15-50, Marcel Dekker, New York, NY, USA, 1996.

[9] C. E. Chidune, J. L. Li, and A. Vdomene, "Convergence of paths and approximation of fixed point of asymptotically non-expansive mapping," Proceedings of the American Mathematical Society, vol. 133, no. 2, pp. 473-480, 2004.

[10] J. Li, "The metric projection and its applications to solving variational inequalities in Banach spaces," Fixed Point Theory, vol. 5, no. 2, pp. 285-298, 2004.

[11] J. Li, "The generalized projection operator on reflexive Banach spaces and its applications," Journal of Mathematical Analysis and Applications, vol. 306, no. 1, pp. 55-71, 2005.

[12] Y. Yao, M. A. Noor, and Y.-C. Liou, "Strong convergence of a modified extragradient method to the minimum-norm solution of variational inequalities," Abstract and Applied Analysis, vol. 2012, Article ID 817436, 9 pages, 2012.

[13] Y.-C. Liou, Y. Yao, C.-W. Tseng, H.-T. Lin, and P.-X. Yang, "Iterative algorithms approach to variational inequalities and fixed point problems," Abstract and Applied Analysis, vol. 2012, Article ID 949141, 15 pages, 2012.

[14] Y. Wang and L. Yang, "Modified relaxed extragradient method for a general system of variational in-equalities and nonexpansive mappings in Banach spaces," Abstract and Applied Analysis, vol. 2012, Article ID 818970, 14 pages, 2012.

[15] Y. Alber and S. Guerre-Delabriere, "Principle of weakly contractive maps in Hilbert spaces," Operator Theory, Advances and Applications, vol. 98, pp. 7-22, 1997.

[16] W. Xu and Y. Wang, "Strong convergence of the iterative methods for hierarchical fixed point problems of an infinite family of strictly nonself pseudocontractions," Abstract and Applied Analysis, vol. 2012, Article ID 457024, 11 pages, 2012. 


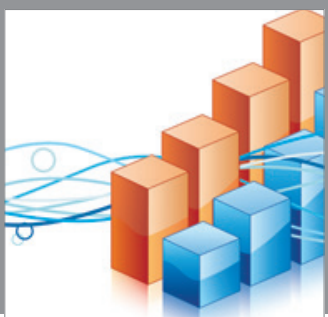

Advances in

Operations Research

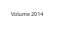

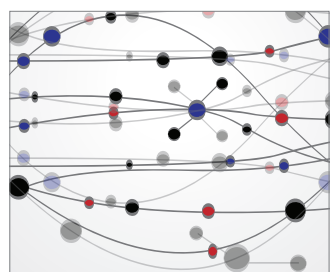

\section{The Scientific} World Journal
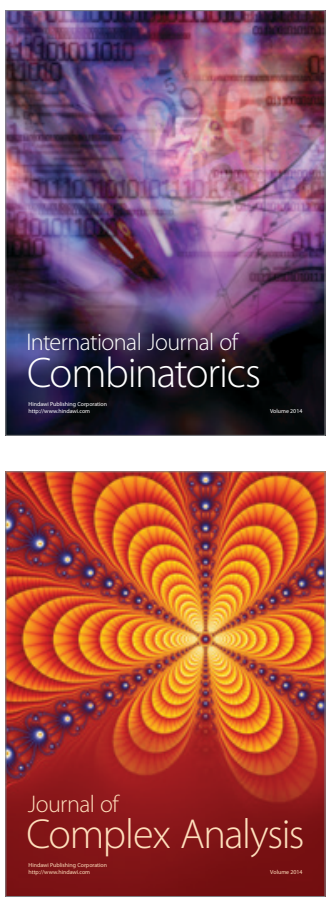

International Journal of

Mathematics and

Mathematical

Sciences
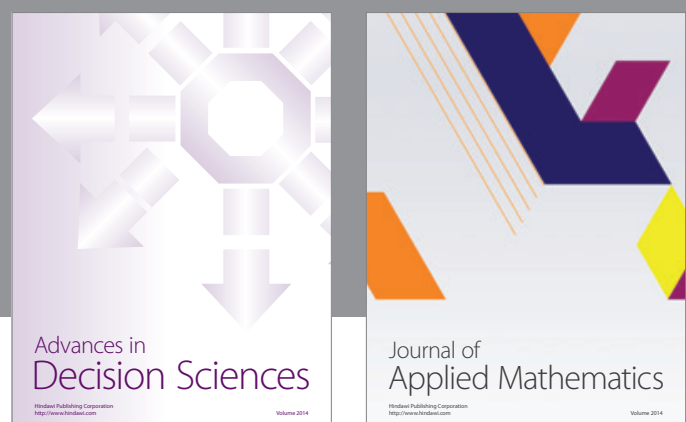

Journal of

Applied Mathematics
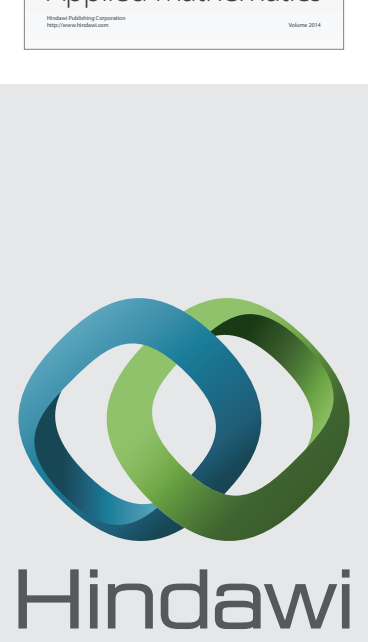

Submit your manuscripts at http://www.hindawi.com
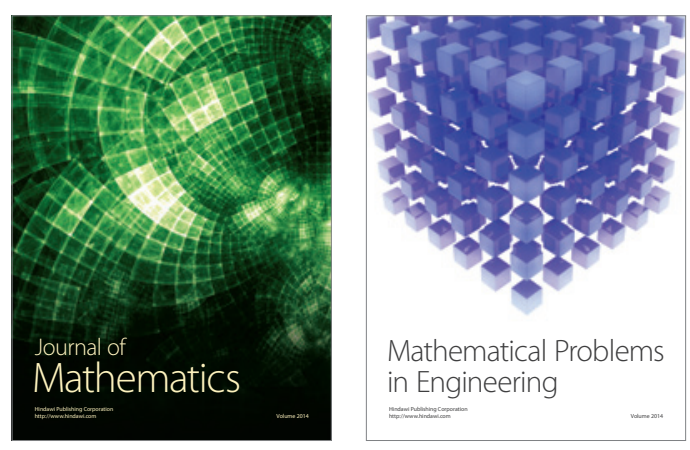

Mathematical Problems in Engineering
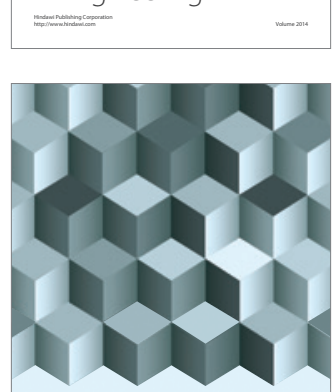

Journal of

Function Spaces
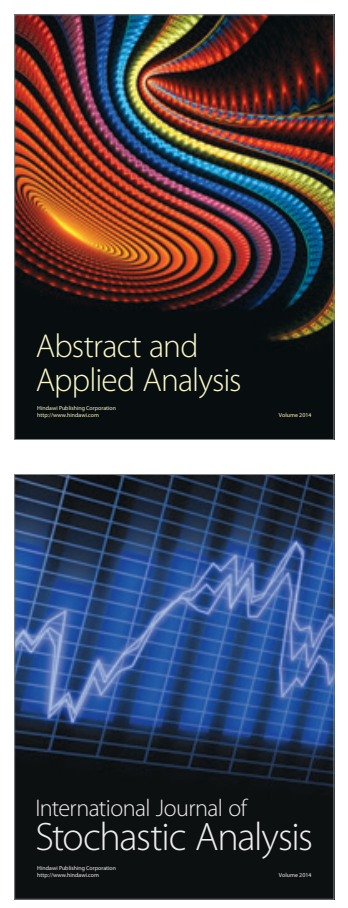

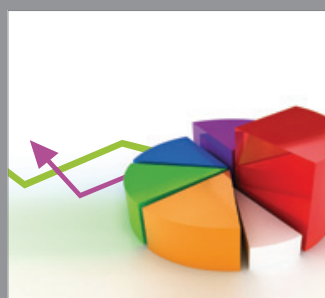

ournal of

Probability and Statistics

Promensencen
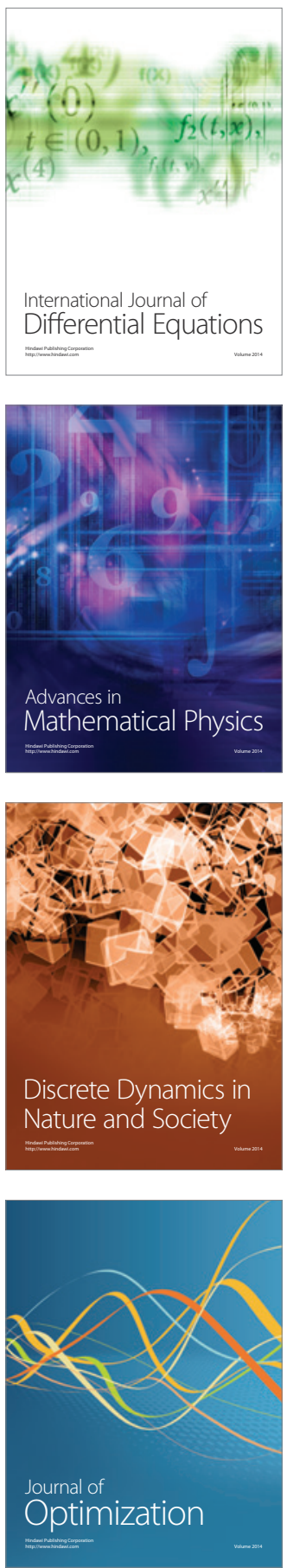